\title{
Topological Transitivity of Shift Similar Operators on Nonseparable Hilbert Spaces
}

\author{
Andriy Zagorodnyuk $\mathbb{D I}^{1}$ and Zoriana Novosad $\mathbb{D}^{2}$ \\ ${ }^{1}$ Vasyl Stefanyk Precarpathian National University, 57 Shevchenka Str., Ivano-Frankivsk 76018, Ukraine \\ ${ }^{2}$ Lviv University of Trade and Economics, 10 Tuhan-Baranovsky Str., Lviv 79005, Ukraine
}

Correspondence should be addressed to Andriy Zagorodnyuk; azagorodn@gmail.com

Received 31 July 2021; Accepted 27 September 2021; Published 18 October 2021

Academic Editor: Ismat Beg

Copyright ( 2021 Andriy Zagorodnyuk and Zoriana Novosad. This is an open access article distributed under the Creative Commons Attribution License, which permits unrestricted use, distribution, and reproduction in any medium, provided the original work is properly cited.

In this paper, we investigate topological transitivity of operators on nonseparable Hilbert spaces which are similar to backward weighted shifts. In particular, we show that abstract differential operators and dual operators to operators of multiplication in graded Hilbert spaces are similar to backward weighted shift operators.

\section{Introduction}

Let $X$ be a Hausdorff locally convex space. A continuous operator $T: X \longrightarrow X$ is called topologically transitive if for each pair $U, V$ of nonempty open subsets of $X$, there is some $n \in \mathbb{N}$ with $T^{n}(U) \cap V \neq \varnothing$. If the underlying space is a separable Baire space, the transitivity is equivalent to the hypercyclicity by Birkhoff's transitivity theorem (see [1], p. 2). A continuous linear operator $T: X \longrightarrow X$ acting on a separable Fréchet space $X$ is called hypercyclic if there is a vector $x \in X$ for which the orbit under $T$,

$$
\operatorname{Orb}(T, x)=\left\{x, T x, T^{2} x, \cdots\right\}
$$

is dense in $X$. Every such vector $x$ is called a hypercyclic vector of $T$.

The study of topological transitivity was started from Birkhoff's result [2] in 1929 for nonlinear continuous functions. This result is important in the theory of chaos (see e.g., [3]). For the space of all entire functions, Birkhoff [2] also proved that the translation operator $T_{a}: f(x) \mapsto f(x+a)$, $(a \neq 0)$ is hypercyclic. MacLane in 1952 showed that the differentiation operator $T(f)=f^{\prime}$ is hypercyclic too (see [4]).

A lot of work on hypercyclicity has been based on the well-known so-called hypercyclicity criterion (the KitaiGethner-Shapiro theorem). In [5], Bermúdez and Kalton observed that similar criterion holds for topologically transitive operators on Banach spaces.

Theorem 1 (topologically transitive criterion). Let $T$ be a bounded linear operator on a complex Banach space X (not necessarily separable). Suppose that there exists a strictly increasing sequence $\left\{n_{k}\right\}$ of positive integers for which there are

(i) A dense subset $X_{0} \subset X$ such that $T^{n_{k}}(x) \longrightarrow 0$ for every $x \in X_{0}$ as $k \longrightarrow \infty$

(ii) A dense subset $Y_{0} \subset X$ and a sequence of mappings $S_{k}: Y_{0} \longrightarrow X$ such that $S_{k}(y) \longrightarrow 0$ for every $y \in Y_{0}$ and $T^{n_{k}} \circ S_{k}(y) \longrightarrow y$ for every $y \in Y_{0}$ as $k \longrightarrow \infty$

Then, $T$ is topologically transitive.

More information on transitive and hypercyclic operators can be found in $[1,6]$. The existence of an element with a dense orbit implies that $X$ must be separable. On the other hand, the transitivity of $T: X \longrightarrow X$ does not require that the space $X$ is separable.

Problem 2 (Bermúdez and Kalton [5]). Is there any characterization of nonseparable Banach spaces which support a topologically transitive operator? 
Note that any hypercyclic operator $T: X \longrightarrow X$ always has an invariant, norm dense, linear subspace in which every nonzero vector is a hypercyclic vector for $T$.

Theorem 3 (see [7]). Let $X$ be a complete metric space which has no isolated points. Let $T: X \longrightarrow X$ be topologically transitive operator and $y \in X$. There exists a dense $G_{\delta}$ subset $D$ of $X$ such that for each $z \in D$, there exists a T-invariant (separable) closed subspace $Y_{z}$ of $X$ with $y, z \in Y_{z}$ such that the restriction of $T$ to $Y_{z}, T: Y_{z} \longrightarrow Y_{z}$, is hypercyclic.

The hypercyclisity of composition operators and differentiation operators on some function spaces of finite and infinite variables was studied in [8-14]. According to a classical theorem of Rolewicz [15], the weighted backward shifts on $\ell_{2}$

$$
\left(x_{1}, x_{2}, \cdots, x_{n}, \cdots\right) \mapsto\left(\lambda x_{2}, \lambda x_{3}, \cdots, \lambda x_{n+1}, \cdots\right)
$$

is hypercyclic if $|\lambda|>1$. This fact remains to be true if we replace $\ell_{2}$ into $\ell_{p}$ for $1 \leq p<\infty$. Bermúdez and Kalton in [5] showed that spaces like $\ell_{\infty}$ and $\mathscr{L}\left(\ell_{2}\right)$ ) do not support topologically transitive operators, where $\mathscr{L}\left(\ell_{2}\right)$ is the space of all bounded operators on $\ell_{2}$.

In [7] (Proposition 3.4), Manoussos proved that the backward weighted shift is topologically transitive on nonseparable Hilbert space. More detailed, let $H$ be a Hilbert space (possibly nonseparable) and $\ell_{2}(H)$ be the $\ell_{2}$-sum of infinitely many copies of $H$. That is, if $x \in \ell_{2}(H)$, then

$$
x=\left(x_{0}, x_{1}, \cdots, x_{n}, \cdots\right), \quad x_{n} \in H,\|x\|^{2}=\sum_{n=0}^{\infty}\left\|x_{n}\right\|^{2} .
$$

Then, the backward weighted shift $T$ with weight sequence $\left(\omega_{n}\right)$ is defined by the following:

$$
T:\left(x_{0}, x_{1}, \cdots, x_{n}, \cdots\right) \mapsto\left(\omega_{1} x_{1}, \omega_{2} x_{2}, \cdots, \omega_{n} x_{n}, \cdots\right)
$$

Unfortunately, Proposition 3.4 in [7] contains a slight inaccuracy which for us is essential. So, we propose the corrected version.

Proposition 4. Let $H$ be a Hilbert space and $T: \ell_{2}(H) \longrightarrow$ $\ell_{2}(H)$ be a backward weighted shift with positive weight sequence $\left(\omega_{n}\right)$. The following are equivalent:

(i) $T$ is topologically transitive

(ii) There exists a nontrivial T-invariant (separable) closed subspace $\mathscr{Y} \subset \ell_{2}(H)$ on which the restriction of $T$ to $\mathcal{Y}, T: \mathcal{Y} \longrightarrow \mathcal{Y}$, is hypercyclic

(iii) The restriction $T: \mathcal{Y} \longrightarrow \mathcal{Y}$ to any $T$-invariant (separable) closed subspace $\mathcal{Y} \subset \ell_{2}(H)$ which contains nonzero vectors of the form $\left(0, \cdots, 0, x_{n}, 0 \cdots\right)$ for every $n \in \mathbb{Z}_{+}$is hypercyclic

(iv) $\limsup _{n \rightarrow \infty} \prod_{k=1}^{n} \omega_{n}=+\infty$
Note that in [7], item (iii) is written: "The restriction $T: \mathcal{Y} \longrightarrow \mathcal{Y}$ to any $T$-invariant (separable) closed subspace $\mathscr{Y} \subset \ell_{2}(H)$ is hypercyclic." But it is not correct because the subspace $Y_{0}$ consisting of vectors $\left(x_{0}, 0,0, \cdots\right), x_{0} \in H$ is invariant but the restriction of $T$ to any separable subspace of $Y_{0}$ is not hypercyclic since $Y_{0} \subset \operatorname{ker} T$.

In Section 2, we consider abstract shift similar operators on nonseparable function Hilbert spaces $\ell_{2}(H)$. In particular, abstract differentiation operators and dual operators to abstract multiplication operators can be considered as abstract shift similar operators. In Section 3, we construct examples of topologically transitive operators.

\section{Abstract Shift Similar Operators}

Let $\left(H_{n}\right)_{n=0}^{\infty}$ be a sequence of Hilbert spaces. Throughout this paper, we assume that all $H_{n}$ are nontrivial; that is, $H_{n} \neq\{0\}$ and not necessary separable. Let us suppose that for every $n$ and $m, H_{n}$ is isomorphic to $H_{m}$. We denote by $\ell_{2}\left(H_{n}\right)$ $=\ell_{2}\left(\left(H_{n}\right)_{n=0}^{\infty}\right)$ the Hilbert space consisting of elements $x=\left(x_{0}, x_{1}, \cdots, x_{n}, \cdots\right), x_{k} \in H_{k}$ endowed with norm $\|x\|=$ $\left(\sum_{i=0}^{\infty}\left\|x_{i}\right\|^{2}\right)^{1 / 2}$. Let $\left(\omega_{n}\right)$ be a sequence of positive numbers (weights). Let us fix a sequence of isomorphisms $J_{m}: H_{m}$ $\longrightarrow H_{m-1},\left\|J_{m}\right\|=1, m \in \mathbb{N}$. An operator $T: \ell_{2}\left(H_{n}\right) \longrightarrow \ell_{2}$ $\left(H_{n}\right)$ will be called a backward weighted shift (with respect to the family $\left(J_{m}\right)$ ) with weight sequence $\left(\omega_{n}\right)$ if it is of the following form:

$$
T(x)=\left(\omega_{1} J_{1}\left(x_{1}\right), \omega_{2} J_{2}\left(x_{2}\right), \cdots, \omega_{m} J_{m}\left(x_{m}\right), \cdots\right) .
$$

From Proposition 4, it follows the next corollary.

Corollary 5. Let $\left(H_{n}\right)_{n=0}^{\infty}$ be a sequence of Hilbert spaces and $T: \ell_{2}\left(H_{n}\right) \longrightarrow \ell_{2}\left(H_{n}\right)$ be a backward weighted shift with respect to $\left(J_{m}\right)$ and with positive weight sequence $\left(\omega_{n}\right)$. Let us suppose that

$$
\sup _{m \in \mathbb{Z}_{+}} \prod_{n=0}^{m}\left\|J_{n}^{-1}\right\|<\infty .
$$

Then, the following are equivalent:

(i) $T$ is topologically transitive

(ii) There exists a nontrivial T-invariant (separable) closed subspace $\mathcal{Y} \subset \mathrm{l}_{2}\left(H_{n}\right)$ on which the restriction of $T$ to $\mathcal{Y}, T: \mathcal{Y} \longrightarrow \mathcal{Y}$, is hypercyclic

(iii) The restriction $T: \mathcal{Y} \longrightarrow \mathcal{Y}$ to any $T$-invariant (separable) closed subspace $y \subset \ell_{2}\left(H_{n}\right)$ which contains nonzero vectors of the form $\left(0, \cdots, 0, x_{n}, 0, \cdots\right)$ ,$x_{n} \in H_{n}$ for every $n \in \mathbb{Z}_{+}$, is hypercyclic

(iv) $\limsup _{n \rightarrow \infty} \prod_{k=1}^{n} \omega_{k}=\infty$ 
Proof. We set $H=H_{0}$. Then,

$$
\begin{aligned}
J_{1}\left(H_{1}\right) & =H_{0}=H, \\
\left(J_{1} \circ J_{2}\right)\left(H_{2}\right) & =H_{0}=H, \\
\left(J_{1} \circ \cdots \circ J_{n}\right)\left(H_{n}\right) & =H_{0}=H,
\end{aligned}
$$

and so on. For any $x=\left(x_{1}, \cdots, x_{n}, \cdots\right) \in \ell_{2}\left(H_{n}\right)$,

$$
\begin{gathered}
J: x \mapsto\left(x_{0}, J_{1}\left(x_{1}\right), \cdots,\left(J_{1} \circ \cdots \circ J_{n}\right)\left(x_{n}\right), \cdots\right) \in \ell_{2}(H), \\
\|J(x)\| \leq\|x\| .
\end{gathered}
$$

Because of Equation (6), the inverse operator

$J^{-1}: z \mapsto\left(z_{0}, J_{1}^{-1}\left(z_{1}\right), \cdots,\left(J_{n}^{-1} \circ \cdots \circ J_{1}^{-1}\right)\left(z_{n}\right), \cdots\right) \in \ell_{2}\left(H_{n}\right), \quad z \in \ell_{2}(H)$

is well defined and bounded. So, $J$ is an isometric isomorphism from $\ell_{2}\left(H_{n}\right)$ to $\ell_{2}(H)$. For a closed subspace $y \subset \ell_{2}$ $\left(H_{n}\right)$, the range $J(\mathcal{Y})$ is a closed subspace of $\ell_{2}(H)$. Let $T^{\prime}=J \circ T \circ J^{-1}$. Then, $T^{\prime}$ is a backward weighted shift on $\ell_{2}(H)$ with positive weight sequence $\left(\omega_{n}\right)$. Since $J$ is an isomorphism, the topological transitivity of $T$ or hypercyclicity of $T$ on $\mathcal{Y}$ is equivalent to the topological transitivity of $T^{\prime}$ on $\ell_{2}(H)$, or hypercyclicity of $T^{\prime}$ on $J(\mathcal{Y})$, respectively. Hence, we can apply Theorem 3 for $T^{\prime}$.

Note that the case when $\left\|J_{n}\right\|=v_{n}>0$ and $\sup _{n} v_{n}<\infty$ can be reduced to our case if we consider $J_{n}^{\prime}=J_{n} / \nu_{n}$ and $\omega_{n}^{\prime}$ $=\omega_{n} \nu_{n}$ instead of $J_{n}$ and $\omega_{n}, n \in \mathbb{N}$, respectively.

An operator $B: \ell_{2}\left(H_{n}\right) \longrightarrow \ell_{2}\left(H_{n}\right)$ is a backward weighted shift similar operator if there exists an isomorphism $A: \ell_{2}\left(H_{n}\right) \longrightarrow \ell_{2}\left(E_{n}\right)$ for some sequence of Hilbert spaces $\left(E_{n}\right)$ and a backward weighted shift $T: \ell_{2}\left(E_{n}\right) \longrightarrow \ell_{2}\left(E_{n}\right)$ such that $B=A T A^{-1}$. It is clear that $B$ is topologically transitive if and only if $T$ is topologically transitive.

Let us suppose that $H_{n}=H_{n}{ }^{\prime} \oplus H_{n}^{0}, n \in \mathbb{Z}_{+}$and the isomorphisms $J_{n}: H_{n} \longrightarrow H_{n-1}$, be such that $J_{n}$ is an isometric isomorphism of $H_{n}{ }^{\prime}$ onto $H_{n-1}{ }^{\prime}$ and $J_{n}$ maps $H_{n}^{0}$ onto $H_{n-1}^{0}$. Also, we denote by $\pi_{n}$ the projection of $H_{n}$ onto $H_{n}{ }^{\prime}$. Then, we define a backward weighted partial shift (with respect to family $\left.\left(J_{m} \circ \pi_{m}\right)\right)$ with weight sequence $\left(\omega_{n}\right)$ on $\ell_{2}\left(H_{n}\right)$ by the following:

$$
T(x)=\left(\omega_{1}\left(J_{1} \circ \pi_{1}\right)\left(x_{1}\right), \omega_{2}\left(J_{2} \circ \pi_{2}\right)\left(x_{2}\right), \cdots, \omega_{m}\left(J_{m} \circ \pi_{m}\right)\left(x_{m}\right), \cdots\right) .
$$

In other words, $\ell_{2}\left(H_{n}\right)=\ell_{2}\left(H_{n}^{\prime}\right) \oplus \ell_{2}\left(H_{n}^{0}\right)$, and $T$ is a backward weighted shift on $\ell_{2}\left(H_{n}^{\prime}\right)$ and ker $T=\ell_{2}\left(H_{n}^{0}\right)$.

Let us recall that the density $d(X)$ of a metric space $X$ is the smallest cardinality of a dense subset of $X$. It is well known that two infinite dimensional Hilbert spaces $H$ and $E$ are isometrically isomorphic if and only if $d(H)=d(E)$. The next proposition shows that the backward weighted partial shift can be reduced to the weighted shift.
Proposition 6. Let $T$ be a backward weighted partial shift with respect to the family of operators $\left(J_{m} \circ \pi_{m}\right)_{m=1}^{\infty}$ with weight sequence $\left(\omega_{n}\right)$ on $\ell_{2}\left(H_{n}\right)$. Let us suppose that all $H_{n}$ are infinite dimensional for $n>1$ and $d\left(H_{n}^{0}\right) \leq d\left(H_{n}\right)$. Then, $T$ is a backward weighted shift similar operator; that is, there is a sequence of Hilbert spaces $\left(E_{n}\right)_{n=0}^{\infty}$ and an isometric isomorphism $\mathscr{I}: \ell_{2}\left(H_{n}\right) \longrightarrow \ell_{2}\left(E_{n}\right)$ such that $\mathscr{I} \circ T \circ \mathscr{I}^{-1}$ is a backward weighted shift.

Proof. Let us define spaces $E_{n}$ by the following way:

$$
\begin{aligned}
& E_{0}=H_{0} \oplus H_{1}^{0} \oplus H_{2}^{0} \oplus \cdots \oplus H_{m}^{0} \oplus \cdots, \\
& E_{n}=H_{n}{ }^{\prime}, \quad n>1 .
\end{aligned}
$$

Since spaces $H_{n}$ are isomorphic each to others, $d\left(H_{n}\right)$ $=d\left(H_{m}\right)$ for all $n, m \in \mathbb{Z}_{+}$. Since $d\left(H_{n}^{0}\right) \leq d\left(H_{n}\right)$, we have $d\left(E_{n}\right)=d\left(E_{m}\right)$ and so $E_{n}$ and $E_{m}$ are isomorphic for all $n$, $m \in \mathbb{Z}_{+}$. It is clear that $\ell_{2}\left(H_{n}\right)$ and $\ell_{2}\left(E_{n}\right)$ are just different representations of the same space, and so $\mathscr{I}$ is the identical operator. Also, the restrictions of $J_{m} \circ \pi_{m}$ to $E_{m}, m>1$ are isometric isomorphisms. So, $\mathscr{I} \circ T \circ \mathscr{I}^{-1}$ is a backward weighted shift with weight sequence $\left(\omega_{n}\right)$ on $\ell_{2}\left(E_{n}\right)$.

Corollary 7. Let $T: \ell_{2}\left(H_{n}\right) \longrightarrow \ell_{2}\left(H_{n}\right)$ be a backward weighted partial shift with positive weight sequence $\left(\omega_{n}\right)$ which satisfies conditions of Proposition 6. Then, the following are equivalent:

(i) $T$ is topologically transitive

(ii) There exists a nontrivial T-invariant (separable) closed subspace $\mathscr{Y} \subset \ell_{2}\left(\left(H_{n}\right)_{n=0}^{\infty}\right)$ on which the restriction of $T$ to $\mathcal{Y}, T: \mathcal{Y} \longrightarrow \mathcal{Y}$, is hypercyclic

(iii) The restriction $T: \mathscr{Y} \longrightarrow \mathcal{Y}$ to any $T$-invariant (separable) closed subspace $\mathcal{Y} \subset \ell_{2}\left(\left(H_{n}\right)_{n=0}^{\infty}\right)$ which contains nonzero vectors of the form $\left(0, \cdots, 0, x_{n}, 0\right.$ $\cdots), x_{n} \in H_{n}{ }^{\prime}$ for every $n \in \mathbb{Z}_{+}$, is hypercyclic

(iv) $\limsup _{n \longrightarrow \infty} \prod_{k=1}^{n} \omega_{k}=\infty$.

This approach can be generalised by the following way.

Theorem 8. Let $D$ be a surjective bounded operator on a $\ell_{2}$ -sum $\ell_{2}\left(\mathscr{H}_{n}\right)$ of Hilbert spaces $\mathscr{H}_{n}$ such that $D: H_{n} \longrightarrow$ $H_{n-1}, n \geq 0$. Then, $D$ is a backward weighted shift similar operator with some weight sequence $\left(\omega_{n}\right), 0<\omega_{n} \leq\left\|D_{n}\right\|$, where $D_{n}$ is the restriction of $D$ to $\mathscr{H}_{n}$.

Proof. Let us define the following spaces:

$\mathscr{H}_{0}^{0}=\mathscr{H}_{0}$,

$\mathscr{H}_{1}^{0}=\operatorname{ker} D_{1}, \mathscr{H}_{1}^{1}=\mathscr{H}_{1} \ominus \operatorname{ker} D_{1}$,

$\mathscr{H}_{2}^{0}=\operatorname{ker} D_{2}, \mathscr{H}_{2}^{1}=\operatorname{ker}\left(D_{1} \circ D_{2}\right) \ominus \mathscr{H}_{2}^{0}, \mathscr{H}_{2}^{2}=\mathscr{H}_{2} \ominus \operatorname{ker}\left(D_{1} \circ D_{2}\right)$, 


$$
\begin{aligned}
\mathscr{H}_{n}^{0} & =\operatorname{ker} D_{n}, \cdots, \mathscr{H}_{n}^{k}=\operatorname{ker}\left(D_{k} \circ \cdots \circ D_{n-1} \circ D_{n}\right) \ominus \mathscr{H}_{n}^{k-1}, \cdots, \\
\mathscr{H}_{n}^{n} & =\mathscr{H}_{n} \ominus \operatorname{ker}\left(D_{1} \circ \cdots \circ D_{n-1} \circ D_{n}\right) .
\end{aligned}
$$

Let us denote by the following:

$$
H_{k}=\ell_{2}\left(\mathscr{H}_{n}^{k}\right)_{n \geq k}=\mathscr{H}_{k}^{k} \oplus \mathscr{H}_{k+1}^{k} \oplus \cdots, \quad k \in \mathbb{Z}_{+} .
$$

In other words, if $0 \neq x \in H_{k}$, then $0 \neq D_{1} \circ \cdots \circ D_{k}(x) \in$ $\mathscr{H}_{0}$. Let $D_{n}^{k}$ be the restriction of $D_{n}$ to $\mathscr{H}_{n}^{k}$. Then, $\left\|D_{n}^{k}\right\| \leq \|$ $D_{n}\|\leq\| D \|, k \leq n$. Thus, the action of $D$ on $H_{k}$ can be written by the following:

$$
D_{(k)}:=D_{0}^{k} \oplus D_{1}^{k} \oplus \cdots \oplus D_{n}^{k} \oplus \cdots, \quad k \in \mathbb{Z}_{+}
$$

and $\left\|D_{(k)}\right\|=\sup _{n}\left\|D_{n}^{k}\right\|$. So, $\left\|D_{n}^{k}\right\| \leq\left\|D_{(k)}\right\| \leq\|D\|, k \leq n$. It is clear that the spaces $\ell_{2}\left(\mathscr{H}_{n}\right)$ and $\ell_{2}\left(H_{n}\right)$ consist of the same vectors and the identity map $\mathfrak{I}: \ell_{2}\left(\mathscr{H}_{n}\right) \longrightarrow \ell_{2}\left(H_{n}\right)$ is an isometric isomorphism. Thus, $D_{(k)}$ is the restriction of $\mathfrak{I} D$ $\mathfrak{\Im}^{-1}$ to $H_{k}$. By the construction, $D_{(k)}: H_{k} \longrightarrow H_{k-1}, k>0$ and $D_{(0)}=0$. Moreover, since ker $\mathfrak{\Im} D \mathfrak{I}^{-1}=H_{0}, \operatorname{ker} D_{(k)}=0$ if $k>0$. In addition, since $D$ is onto, $D_{(k)}$ must be onto for every $k$, because the preimage of $H_{k-1}$ under $D_{k}, k>0$ is $H_{k}$. Hence, every $D_{(k)}, k>0$ is an isomorphism.

Let us set the following:

$$
\begin{aligned}
J_{k} & :=\frac{D_{k}}{\left\|D_{k}\right\|}, \\
\omega_{k} & =\left\|D_{k}\right\|, \quad \text { for } k>1 .
\end{aligned}
$$

Then for every $x=\left(x_{0}, x_{1}, \cdots, x_{n}, \cdots\right) \in \ell_{2}\left(H_{n}\right)$,

$$
\mathfrak{J} D \mathfrak{I}^{-1}(x)=\left(\omega_{1} J_{1}\left(x_{1}\right), \omega_{2} J_{2}\left(x_{2}\right), \cdots, \omega_{n} J_{n}\left(x_{n}\right), \cdots\right),
$$

that is, $\mathfrak{\Im} D \mathfrak{\Im}^{-1}$ is a backward weighted shift.

Let us show that $\omega_{k}>0$ for every $k \in \mathbb{N}$. Since all spaces $\mathscr{H}_{k}$ are nontrivial and $D$ is onto, there is a vector $a_{1} \in \mathscr{H}_{1}$ such that $a_{1} \notin \operatorname{ker} D$. So, $a_{1} \in H_{1}$ and $D_{(1)}\left(a_{1}\right)=D_{1}\left(a_{1}\right) \neq 0$; that is, $\omega_{1}=\left\|D_{(1)}\right\| \neq 0$. If already we have nonzero elements $a_{j} \in H_{j} \cap \mathscr{H}_{j}, j=1,2, \cdots, k-1$, then we denote by $a_{k}$ a vector in $\mathscr{H}_{k}$ in the preimage of $a_{k-1}$ under $D_{k}$. By the assumption $a_{k-1} \notin \operatorname{ker}\left(D_{1} \circ \cdots \circ D_{k-1}\right)$, so $a_{k} \notin \operatorname{ker}\left(D_{1} \circ \cdots \circ D_{k}\right)$. Thus, $a_{k} \in$ $H_{k}$ and $D_{(k)}\left(a_{k}\right)=D_{k}\left(a_{k}\right)=a_{k-1} \neq 0$. Hence, $\omega_{k}=\left\|D_{(k)}\right\| \neq 0$.

An element $x \in \ell_{2}\left(\mathscr{H}_{n}\right)$ is a finite type vector if there is $m \in \mathbb{N}$ such that $x_{k}=0$ for all $k \geq m$.

Definition 9. Let $\mathscr{H}_{n}, n \in \mathbb{Z}_{+}$be the Hilbert spaces. We say that $\ell_{2}\left(\mathscr{H}_{n}\right)$ is a graded Hilbert space, if there is a bilinear map $(x, y) \mapsto x y$ (multiplication) defined for every finite type vector $x$ and an arbitrary $y \in \ell_{2}\left(\mathscr{H}_{n}\right)$ with values in $\ell_{2}\left(\mathscr{H}_{n}\right)$ such that the multiplication is associative, nondegenerated (that is, $x y=0$ only if $x=0$ or $y=0$ ) and $x_{n} y_{m} \in \mathscr{H}_{n+m}$ for every $x_{n} \in \mathscr{H}_{n}$ and $y_{m} \in \mathscr{H}_{m}$.
Definition 10. For a given graded Hilbert space $\ell_{2}\left(\mathscr{H}_{n}\right)$ and a finite type vector $a \in \ell_{2}\left(\mathscr{H}_{n}\right)$, we denote by $M_{a}$ the multiplication operator.

$$
M_{a}(x)=a x, \quad x \in \ell_{2}\left(\mathscr{H}_{n}\right) .
$$

Theorem 11. Let $a \in \mathscr{H}_{m}$ and $M_{a}$ be continuous on $\ell_{2}\left(\mathscr{H}_{n}\right)$. If the dual operator $M_{a}^{*}:\left(\ell_{2}\left(\mathscr{H}_{n}\right)\right)^{*}=\ell_{2}\left(\mathscr{H}_{n}^{*}\right) \longrightarrow \ell_{2}\left(\mathscr{H}_{n}^{*}\right)$ is surjective, then it is a backward weighted shift similar operator.

Proof. Let

$$
E_{0}=\mathscr{H}_{0} \oplus \cdots \oplus \mathscr{H}_{m-1}, \cdots, E_{k}=\mathscr{H}_{k m} \oplus \cdots \oplus \mathscr{H}_{k m+k-1}, \cdots
$$

and $H_{n}=E_{n}^{*}$ be the Hermitian dual space for every $n \in \mathbb{Z}_{+}$. Then, $M_{a}$ maps $E_{n}$ to $E_{n+1}, n \in \mathbb{Z}_{+}$and $M_{a}^{*}$ maps $H_{n}$ to $H_{n-1}, n>0$, and $H_{0}=\operatorname{ker} M_{a}^{*}$. Let $\left(M_{a}^{*}\right)_{n}$ be the restriction of $M_{a}^{*}$ to $H_{n}$. Since $M_{a}$ is continuous, $M_{a}^{*}$ is continuous on $\ell_{2}\left(H_{n}\right)$ as well and $\sup _{n}\left\|\left(M_{a}^{*}\right)_{n}\right\|<\infty$. We set the following:

$$
\begin{gathered}
J_{n}=\frac{\left(M_{a}^{*}\right)_{n}}{\left\|\left(M_{a}^{*}\right)_{n}\right\|}, \\
\omega_{n}=\left\|\left(M_{a}^{*}\right)_{n}\right\| .
\end{gathered}
$$

Since $M_{a}^{*}$ is surjective, $\left(M_{a}^{*}\right)_{n}$ are surjective for all $n \in \mathbb{Z}_{+}$ and since $H_{0}=\operatorname{ker} M_{a}^{*},\left(M_{a}^{*}\right)_{n}$ are bijective for $n>0$. So,

$M_{a}^{*}\left(x_{0}, x_{1}, \cdots, x_{k}, \cdots\right)=\left(\omega_{1} J_{1}\left(x_{1}\right), \omega_{2} J_{2}\left(x_{2}\right), \cdots, \omega_{k} J_{k}\left(x_{k}\right), \cdots\right)$

is a backward weighted shift on $\ell_{2}\left(H_{n}\right)$.

Note that if $a \in \mathscr{H}_{1}$, then $E_{k}=\mathscr{H}_{k}$ and $\omega_{n}=\left\|\left(M_{a}^{*}\right)_{n}\right\|=$ $\left\|\left(M_{a}\right)_{n-1},\right\|$, where $\left(M_{a}\right)_{k}$ is the restriction of $M_{a}$ to $\mathscr{H}_{k}$, $k \in \mathbb{Z}_{+}$.

Definition 12. Let $\ell_{2}\left(\mathscr{H}_{n}\right)$ be a graded Hilbert space. A continuous linear operator $D: \ell_{2}\left(\mathscr{H}_{n}\right) \longrightarrow \ell_{2}\left(\mathscr{H}_{n}\right)$ is called a differential operator if $\mathscr{H}_{0} \subset \operatorname{ker} D$, the restriction of $D$ to $\mathscr{H}_{n}$ maps $\mathscr{H}_{n}$ onto $\mathscr{H}_{n-1}, n>1$ and

$$
D(x y)=D(x) y+x D(y)
$$

for every finite type vector $x$ and for all $y \in \ell_{2}\left(\mathscr{H}_{n}\right)$.

Corollary 13. Let $D$ be a differential operator on a graded Hilbert space $\ell_{2}\left(\mathscr{H}_{n}\right)$ such that $D$ is onto and $\left\|D_{1}\right\| \neq 0$. Then, there is a sequence of Hilbert spaces $\left(H_{n}\right)_{n=0}^{\infty}$ and an isometric isomorphism $\mathfrak{I}: \ell_{2}\left(\mathscr{H}_{n}\right) \longrightarrow \ell_{2}\left(H_{n}\right)$ such that $\mathfrak{I} \circ D \circ \mathfrak{J}^{-1}$ is a backward weighted shift with some weight sequence $\left(\omega_{n}\right)$, $0<\omega_{n} \leq\left\|D_{n}\right\|$. 
Proof. Since $\left\|D_{1}\right\| \neq 0$, there is $a \in \mathscr{H}_{1}$ such that $D(a) \neq 0$. So, $a \in H_{1}$. Moreover, for every $k \in \mathbb{N}, a^{k}=a \cdots a \neq 0$ and $D\left(a^{k}\right)$<smiles>CC(C)C(C)C</smiles>

$=k a^{k-1}$. Thus, $a^{k} \in H_{k}$ and we can apply Theorem 2.4.

Corollary 14. Let $D$ be a continuous surjective differential operator on a graded Hilbert space $\ell_{2}\left(\mathscr{H}_{n}\right)$. The following are equivalent:

(i) $D$ is topologically transitive

(ii) There exist $a \in \mathscr{H}_{1}, \varepsilon>0$, and $m \in \mathbb{N}$ such that $D(a)$ $\neq 0$ and

$$
\limsup _{m \rightarrow \infty} \prod_{n=1}^{m} \frac{n\left\|a^{n-1}\right\|}{\left\|a^{n}\right\|}=\infty
$$

(iii)

$$
\underset{m \rightarrow \infty}{\limsup } \prod_{n=1}^{m}\left\|D_{(n)}\right\|=\infty
$$

Proof. As we observed, $a^{\mathrm{k}} \in H_{k}$ and so

$$
\omega_{k}=\left\|D_{(k)}\right\| \geq \frac{\left\|D_{(k)}\left(a^{k}\right)\right\|}{\left\|a^{k}\right\|}=\frac{k\left\|a^{k-1}\right\|}{\left\|a^{k}\right\|} .
$$

By Theorem 8., $\omega_{k}>0, k \in \mathbb{N}$. So if $\omega_{k}>1+\varepsilon$ for all $k>$ $m$, then

$$
\prod_{n=1}^{\infty} \omega_{n}=\infty
$$

and $D$ must be topologically transitive.

\section{Examples of Topologically Transitive Operators}

3.1. Partial Shifts. It is well known that any element $x$ of the (complete) Hilbert tensor product $\ell_{2} \otimes_{h} \ell_{2}$ can be represented by the following series

$$
x=\sum_{i, j=0}^{\infty} x_{i j} e_{i} \otimes e_{j}, \text { where }\left(e_{i}\right)_{i \in \mathbb{Z}_{+}} \text {is the standard basis in } \ell_{2}
$$

or by the following infinite matrix

$$
x=\left(x_{i j}\right)_{i, j \in \mathbb{Z}_{+}}=\left(\begin{array}{ccccc}
x_{00} & x_{01} & \cdots & x_{0 n} & \cdots \\
x_{10} & x_{11} & \cdots & x_{1 n} & \cdots \\
\cdots & \cdots & \cdots & \cdots & \cdots \\
x_{n 0} & x_{n 1} & \cdots & x_{n n} & \cdots \\
\cdots & \cdots & \cdots & \cdots & \cdots
\end{array}\right)
$$

with $\|x\|^{2}=\sum_{i, j=0}^{\infty}\left|x_{i j}\right|^{2}$. Note that $\ell_{2} \otimes_{h} \ell_{2}$ can be considered as $\ell_{2}\left(\ell_{2}\right)$ by the following representation:

$$
x=\left(x_{0}, x_{1}, \cdots, x_{n}, \cdots\right) \in \ell_{2}\left(\ell_{2}\right),
$$

where

$$
x_{n}=\left(x_{n 0}, x_{n 1}, \cdots, x_{n n}, \cdots\right) \in \ell_{2}, \quad n \in \mathbb{Z}_{+} .
$$

Let $\omega=\left(\omega_{i j}\right)_{i, j}$ be an infinite matrix of positive numbers. We consider the following operators on $\ell_{2}\left(\ell_{2}\right)$. Let

$$
T_{\omega}(x)=\left(\omega_{i+1, j} x_{i+1, j}\right)_{i, j \in \mathbb{Z}_{+}}=\left(\begin{array}{ccccc}
\omega_{10} x_{10} & \omega_{11} x_{11} & \cdots & \omega_{1 n} x_{1 n} & \cdots \\
\omega_{20} x_{20} & \omega_{21} x_{21} & \cdots & \omega_{2 n} x_{2 n} & \cdots \\
\cdots & \cdots & \cdots & \cdots & \cdots \\
\omega_{n 0} x_{n 0} & \omega_{n 1} x_{n 1} & \cdots & \omega_{n n} x_{n n} & \cdots \\
\cdots & \cdots & \cdots & \cdots & \cdots
\end{array}\right)
$$

and $T_{\omega}^{t}$ be the transposed operator, $T_{\omega}(x)=\left(\omega_{i, j+1} x_{i, j+1}\right)_{i, j \in \mathbb{Z}_{+}}$. Let $\omega_{i}=\sup _{j} \omega_{i j}$. From Theorem 8., it follows that if $T_{\omega}$ is continuous and

$$
\limsup _{n \rightarrow \infty} \prod_{i=1}^{n} \omega_{i}=+\infty
$$

then operators $T_{\omega}^{m} T_{\omega}^{t n}$ are topologically transitive for every $m, n, m+1 \geq 0$. In particular, the operator

$$
T_{\omega} T_{\omega}^{t}(x)=\left(\begin{array}{ccccc}
\omega_{11} x_{11} & \omega_{12} x_{12} & \cdots & \omega_{1 n} x_{1 n} & \cdots \\
\omega_{21} x_{21} & \omega_{22} x_{22} & \cdots & \omega_{2 n} x_{2 n} & \cdots \\
\ldots & \ldots & \ldots & \cdots & \cdots \\
\omega_{n 1} x_{n 1} & \omega_{n 2} x_{n 2} & \cdots & \omega_{n n} x_{n n} & \cdots \\
\ldots & \ldots & \ldots & \ldots & \ldots
\end{array}\right)
$$

looks like weighted diagonal shift operator. It is clear that these examples can be generalised for any tensor degree $\otimes_{h}^{m} \ell_{2}$, $m \in \mathbb{N}$. Next, we consider a nonseparable case.

Let $\mathfrak{A}$ be the set of indexes (uncountable, in general) which can be represented as a disjoint union of subsets $\mathfrak{A}_{i}$.

$$
\mathfrak{U}=\coprod_{i=0}^{\infty} \mathfrak{A}_{i},
$$


and there are a sequence of bijections $\tau_{n}: \mathfrak{A}_{n} \longrightarrow \mathfrak{\Re}_{n-1}$, $n \in \mathbb{N}$. So, we can write $\ell_{2}(\mathfrak{A})=\ell_{2}\left(\ell_{2}\left(\mathfrak{A}_{n}\right)\right)$ and define $J_{n}: \ell_{2}\left(\mathfrak{\Re}_{n}\right) \longrightarrow \ell_{2}\left(\mathfrak{A}_{n-1}\right), n \in \mathbb{N}$ by $J_{n}\left(x_{i}\right)=x_{\tau_{n}(i)}, i \in \mathfrak{A}_{n}$. By Corollary 5 , we have that

$$
T(x)=\left(\omega_{1} J_{1}\left(x_{1}\right), \omega_{2} J_{2}\left(x_{2}\right), \cdots, \omega_{m} J_{m}\left(x_{m}\right), \cdots\right)
$$

is topologically transitive if and only if the weight $\left(\omega_{m}\right)$ satisfies Equation (31). Moreover, the Hilbert tensor product $\ell_{2}(\mathfrak{U}) \otimes_{h} \ell_{2}(\mathfrak{\mathfrak { U }})$ is isomorphic to

$$
\ell_{2}(\mathfrak{A} \times \mathfrak{A})=\ell_{2}\left(\ell_{2}\left(\mathfrak{A}_{n} \times \mathfrak{A}_{m}\right)\right)
$$

For a given weight matrix $\omega=\omega_{i, j}, i, j \in \mathbb{Z}_{+}, \omega_{i, j}>0$, we define operators $T_{\omega}$ and $T_{\omega}^{t}$ on $\ell_{2}\left(\ell_{2}\left(\mathfrak{A}_{n} \times \mathfrak{A}_{m}\right)\right)$ by the following:

$$
T_{\omega}\left(x_{n m}\right)=x_{\tau(n) m} \text { and } T_{\omega}^{t}\left(x_{n m}\right)=x_{n \tau(m)},
$$

where $x_{n m} \in \ell_{2}\left(\mathfrak{\mathcal { A }}_{n} \times \mathfrak{\mathfrak { A }}_{m}\right)$.

Corollary 15. Let

$$
\sup _{i, j \in \mathbb{Z}_{+}} \omega_{i, j}<\infty
$$

then for every $k, j \in \mathbb{Z}_{+}, k+j>0$, operators $T_{\omega}^{k} T_{\omega}^{t j}$ are topologically transitive if and only if

$$
\limsup _{n \rightarrow \infty} \prod_{i=1}^{n} \sup _{m} \omega_{m i}=+\infty \text {. }
$$

3.2. Differential Operator on a Function Space. Examples of Hilbert spaces of analytic functions with continuous differential operators can be found among reproducing kernel Hilbert spaces.

Definition 16. Let $Z$ be an abstract set and $\mathscr{H}$ a Hilbert space of complex valued functions $f$ on $Z$ equipped with inner product $\langle\cdot \mid \cdot\rangle_{\mathscr{H}}$. A function $K(x \mid z)$ defined on $Z \times Z$ is called reproducing kernel of a closed subspace $\mathscr{H}_{K} \subset \mathscr{H}$ if

(i) For any fixed $z \in Z, K(x \mid z)$ belongs to $\mathscr{H}_{K}$ as a function in $x \in Z$

(ii) For any $f \in \mathscr{H}_{K}$ and for any $z \in Z, f(z)=\langle f \mid K(\cdot \mid z)\rangle_{\mathscr{H}}$

The space $\mathscr{H}_{K}$ is called a Hilbert space with reproducing kernel. A function $h: Z \longrightarrow \mathscr{H}$ such that

$$
f(x)=\langle f(\cdot) \mid h(x)\rangle_{\mathscr{H}}
$$

for every $f \in \mathscr{H}_{K}$ and $x \in Z$ is called the kernel function of $\mathscr{H}_{K}$.

Let $\mathscr{H}(\gamma)$ be the Hilbert space of power series

$$
f(z)=\sum_{n=0}^{\infty} \widehat{f}(n) z^{n}, z \in \mathbb{C}
$$

endowed with the norm

$$
\|f\|^{2}=\sum_{n=0}^{\infty} \gamma_{n}^{-2}|\widehat{f}(n)|^{2} \leq \infty,
$$

where $\gamma=\left\{\gamma_{n}\right\}$ is a positive sequence and $\widehat{f}(n) \in \mathbb{C}$. So, the inner product on $\mathscr{H}(\gamma)$ is defined by $\langle f \mid g\rangle=\sum_{n=0}^{\infty} \gamma_{n}^{-2} \widehat{f}(n)$ $\hat{g}(n)$ and $\mathscr{H}(\gamma)$ is a reproducing kernel space with the reproducing kernel.

$$
K(x \mid z)=\sum_{n=0}^{\infty} \gamma_{n}^{2} x^{n} \bar{z}^{n} .
$$

It is known [16] that if $\gamma_{n+1} / \gamma_{n} \longrightarrow 0$ as $n \longrightarrow \infty, \mathscr{H}(\gamma)$ consists of entire functions on $\mathbb{C}$.

Let $D=d / d z$ be the differential operator on $\mathscr{H}(\gamma)$.Then, $D_{n}\left(\widehat{f}(n) z^{n}\right)=\widehat{f}(n) n z^{n-1}, n>0$, and so $\left\|D_{n}\right\|=n \gamma_{n} / \gamma_{n-1}$. Thus, $D$ is continuous (c. f. [9]) if and only if

$$
\sup _{n}\left\|D_{n}\right\|=\sup _{n} \frac{n \gamma_{n}}{\gamma_{n-1}}<\infty
$$

On the other hand, according to Corollary $14, \mathrm{D}$ is topologically transitive and so hypercyclic if and only if

$$
\underset{\mathrm{m} \longrightarrow \infty}{\limsup } \prod_{n=1}^{m} \frac{n \gamma_{n}}{\gamma_{n-1}} \longrightarrow \infty \text {. }
$$

It is clear that $\mathscr{H}^{*}(\gamma)=\mathscr{H}(1 / \gamma)$. Let us consider the multiplication operator $M_{z}$ on $\mathscr{H}(\gamma), M_{z}(f)=z f(z)$. Then, $\|$ $\left(M_{z}\right)_{n} \|=\gamma_{n} / \gamma_{n+1}$. According to Theorem 11, $M_{z}$ is bounded if and only if $\sup _{n} \gamma_{n} / \gamma_{n+1}<\infty$ and $M_{\mathrm{z}}$ is topologically transitive if and only if

$$
\underset{m \longrightarrow \infty}{\limsup } \prod_{n=1}^{m} \frac{\gamma_{n}}{\gamma_{n+1}} \longrightarrow \infty \text {. }
$$

This result agrees with [8]. Various Hilbert spaces of analytic functions of infinite many variables were constructed in $[14,17,18]$ using the Hilbertian symmetric tensor products of Hilbert spaces. Such constructions can be easily extended to nonseparable Hilbert spaces. In [14], the authors found some conditions under which the translation operator $f(x) \mapsto f(x+a)$ on a Hilbert space of entire analytic functions on $\ell_{2}$ is hypercyclic. Taking into account that the translation operator can be represented as an exponential function of a differential operator, we would like to ask the following question.

Question 17. Let $D$ be a differential operator on a graded nonseparable Hilbert space. Suppose that $D$ is topologically transitive. Does 


$$
e^{D}=\sum_{n=0}^{\infty} \frac{D^{n}}{n !}
$$

topologically transitive?

\section{Conclusion}

We can see that dual to abstract multiplication operators $M_{a}^{*}$ and abstract differentiation operators $D$ on Hilbert spaces can be represented as backward weighted shift operators. Thus, we can use known results about topological transitivity of backward weighted shift operators to get conditions of topological transitivity of operators $M_{a}^{*}$ and $D$.

\section{Data Availability}

No data were used to support this study.

\section{Conflicts of Interest}

The authors declare that they have no conflicts of interest.

\section{Acknowledgments}

This research was funded by the National Research Foundation of Ukraine (2020.02/0025, 0121U111037).

\section{References}

[1] F. Bayart and E. Matheron, Dynamics of Linear Operators, Cambridge University Press, New York, 2009.

[2] G. D. Birkhoff, "Démonstration d'un théorème élémentaire sur les fonctions entières," C. R. Acad. Sci. Paris, vol. 189, pp. 473475, 1929.

[3] R. L. Devaney, An Introduction to Chaotic Dynamical Systems, Addison-Wesley, Reedwood City, 1989.

[4] G. R. MacLane, "Sequences of derivatives and normal families," Journal d'Analyse Mathématique, vol. 2, no. 1, pp. 72$87,1952 / 53$.

[5] T. Bermúdez and N. J. Kalton, "The range of operators on von Neumann algebras," Proceedings of the American Mathematical Society, vol. 130, no. 5, pp. 1447-1455, 2002.

[6] K. G. Grosse-Erdmann and A. Peris Manguillot, Linear Chaos, Springer-Verlag, London, 2011.

[7] A. Manoussos, "A Birkhoff type transitivity theorem for nonseparable completely metrizable spaces with applications to linear dynamics," Journal of Operator Theory, vol. 70, no. 1, pp. 165-174, 2103.

[8] G. Godefroy and J. H. Shapiro, "Operators with dense, invariant, cyclic vector manifolds," Journal of Functional Analysis, vol. 98, no. 2, pp. 229-269, 1991.

[9] K. C. Chan and J. H. Shapiro, "The cyclic behavior of translation operators on Hilbert spaces of entire functions," Indiana University Mathematics Journal, vol. 40, no. 4, pp. 14211449, 1991.

[10] R. Aron and J. Bès, "Hypercyclic differentiation operators," Contemporary Mathematics, vol. 232, pp. 39-46, 1999.

[11] R. M. Gethner and J. H. Shapiro, "Universal vectors for operators on spaces of holomorphic functions," Proceedings of the
American Mathematical Society, vol. 100, no. 2, pp. 281-288, 1987.

[12] P. S. Bourdon and J. H. Shapiro, "Cyclic phenomena for composition operators," Memoirs of the American Mathematical Society, vol. 125, no. 596, pp. 1-46, 1997.

[13] J. H. Shapiro, Composition Operators and Classical Function Theory, Springer, New York, 1993.

[14] Z. H. Mozhyrovska and A. V. Zagorodnyuk, "Hypercyclic behavior of translation operators on spaces of analytic functions on Hilbert spaces," J. Funct. Spaces, vol. 2015, article 139289, 8 pages, 2015.

[15] S. Rolewicz, "On orbits of elements," Studia Mathematica, vol. 32, no. 1, pp. 17-22, 1969.

[16] S. M. Duyos-Ruiz, "On the existence of universal functions," Soviet Math. Dokl., vol. 27, pp. 9-13, 1983.

[17] O. Lopushansky and A. Zagorodnyuk, "Representing measures and infinite-dimensional holomorphy," Journal of Mathematical Analysis and Applications, vol. 333, no. 2, pp. 614$625,2007$.

[18] O. Lopushansky, "The Hilbert-Schmidt analyticity associated with infinite dimensional unitary groups," Results in Mathematics, vol. 71, no. 1-2, pp. 111-126, 2017. 